\title{
Balkanologie
}

Balkanologie Revue d'études pluridisciplinaires

Vol. III, n 1 | 1999

Volume III Numéro 1

\section{Un aspect de la propagande titiste : le culte de Tito dans le quotidien Politika (1945 à 1980)}

Suzana Đukić

\section{(2) OpenEdition}

12 Journals

Édition électronique

URL : http://journals.openedition.org/balkanologie/287

DOI : 10.4000/balkanologie.287

ISSN : 1965-0582

Éditeur

Association française d'études sur les Balkans (Afebalk)

Édition imprimée

Date de publication : 1 juillet 1999

Pagination : 67-87

ISSN : 1279-7952

\section{Référence électronique}

Suzana Đukić, « Un aspect de la propagande titiste : le culte de Tito dans le quotidien Politika (1945 à 1980) », Balkanologie [En ligne], Vol. III, $n^{\circ} 1$ | 1999, mis en ligne le 30 novembre 2007, consulté le 17 décembre 2020. URL : http://journals.openedition.org/balkanologie/287 ; DOI : https://doi.org/ 10.4000/balkanologie.287

Ce document a été généré automatiquement le 17 décembre 2020.

(c) Tous droits réservés 


\title{
Un aspect de la propagande titiste : le culte de Tito dans le quotidien Politika (1945 à 1980)
}

\author{
Suzana Đukić
}

\section{NOTE DE L'ÉDITEUR}

Cet article a été rédigé à partir du mémoire de maîtrise Le Culte de la Personnalité de Tito de 1945 à 1980, sous la direction de M. F. Rousseau, maître de conférences en Histoire Contemporaine, Université Paul Valéry, Montpellier III.

1 «Posle Tita, Tito ", "Après Tito, Tito ", voilà à quoi se résumait le programme de la classe dirigeante yougoslave à la mort de son prestigieux chef en 1980. Ce slogan, lancé du vivant même de Josip Broz Tito, devait préparer la population à continuer sur la voie $\mathrm{du}$ socialisme yougoslave initié par celui que l'on surnommait affectueusement "Stari" (le Vieux) et qui a dominé le pays de 1945 à 1980. D'après l'historien Joseph Krulić ${ }^{1}$, « le culte de la personnalité a été un outil politique utilisé par le titisme ». C'est ce que nous voulons mesurer par l'étude du traitement accordé par le quotidien Politika à la fête nationale du 25 mai. Cette fête célèbre "l'anniversaire de Tito", puis à partir de 1956, le "Jour de la Jeunesse". De multiples manifestations culturelles et sportives avaient lieu le $25 \mathrm{mai}^{2}$ de chaque année, la principale étant l'arrivée à Belgrade de la štafeta, la course de relais courue à travers tout le pays. Les coureurs transmettaient au cours d'une cérémonie officielle le(s) témoin(s) de la course, place de la République ou à Beli Dvor, et à partir de 1956, au stade de l'Armée Populaire de Yougoslavie (JNA Jugoslovenska Narodna Armija). 


\section{La presse, un outil de propagande utilisé par le titisme}

\section{Politika, un quotidien lié au pouvoir}

2 D'après J-M. Domenach ${ }^{3}$, « l'étatisation ou le contrôle étatique de l'information donnent une supériorité énorme aux propagandes gouvernementales, dans les régimes de parti unique ", d'où l'intérêt d'une étude de presse pour le cas de la Yougoslavie de Tito. Le contrôle de l'information permet la diffusion de messages propagandistes sous formes variées (rubriques) et adaptés au profil sociologique du lecteur. Cette presse applique donc à la fois les lois d'orchestration ${ }^{4}$, c'est-à-dire de répétition sous différentes formes des mêmes messages et de différenciation, d'adaptation des messages en fonction du public auquel on s'adresse.

3 La politique d'information du quotidien du matin Politika ${ }^{5}$ est favorable à Tito, dans la mesure où un fort lien existe entre le pouvoir et la rédaction : un timbre-poste est émis en 1979 pour célébrer l'anniversaire de la création de Politika (en 1904). Cet organe de presse belgradois rédigé en cyrillique n'est pas nécessairement un journal présentant une orientation spécifiquement serbe, puisque Tito a toujours insisté pour que les variante "occidentale" et "orientale" de l'écriture, c'est à dire respectivement les alphabets latin et cyrillique, s'emploient indifféremment, sans connotation religieuse, ethnique ou politique.

\section{Permanences du traitement journalistique}

Les articles concernant la célébration du 25 mai débutent toujours avec un récapitulatif des luttes du Parti communiste et du rôle de Tito dans l'histoire nationale. Invariablement, les articles du 25 mai reprennent l'action du maréchal yougoslave dans le Parti communiste en tant que militant, la direction par le "Vieux" des forces armées pendant le second conflit mondial, ainsi que son œuvre de chef du gouvernement et de président de la République (la réconciliation nationale, l'autogestion à partir de 1950, le non-alignement à partir de 1961). En terme journalistique, on parle d'"article historique", c'est à dire d'articles qui "font revivre le passé à l'occasion d'un anniversaire par exemple $»^{6}$. Cette répétitivité est expliquée par le courant pavlovien de la psychologie sociale comme ayant pour but d'inculquer un réflexe conditionné associant Tito aux grandes étapes de la vie du pays. Les mêmes faits sont systématiquement repris et c'est à l'évolution de la façon dont Tito est présenté que nous nous intéressons. Les pages suivantes du journal sont généralement consacrées à la publication des télégrammes de félicitations envoyés au dirigeant yougoslave et aux descriptions et commentaires de la fin de la course de relais sous formes de reportages. Les journaux du 26 mai nous ont également servi, car ils présentaient un compte rendu des activités de la veille. Des télégrammes de félicitations, des reportages, des témoignages sont toujours publiés au lendemain de la célébration.

5 Le traitement journalistique du 25 mai relève d'une propagande émotionnelle et persuasive ainsi que d'un jeu entre propagande "voilée" et "révélée", d'après les expressions de J. Ellul ${ }^{7}$ :

* la fête du 25 mai relève d'une propagande émotionnelle, d'agitation, c'est-à-dire qu'elle vise à l'excitation des foules. Le spectacle du 25 mai est une manifestation de pouvoir. Ses intentions sont clairement identifiées: il s'agit d'inciter le citoyen à 
reconnaître la valeur de Tito. C'est également un divertissement, un jeu offert à la population, qui est détournée de ses préoccupations quotidiennes pour un temps ;

* les articles de journaux concernant cette date sont une propagande de type persuasive, des démonstrations logiques sont exposées. Les articles panégyriques, les comptes rendus des spectacles, des réceptions, la publication des télégrammes de félicitations dans le quotidien Politika relèvent de la propagande indirecte car le destinataire ne sait pas que l'on cherche à l'influencer. Les journaux préparent et prolongent la célébration.

6 L'État yougoslave, comme tous les États d'une façon générale, considère que la gestion de la mémoire est une de ses prérogatives. La mémoire collective est donc gérée par le sommet. Mais l'État titiste a une intention totalitaire, celle du contrôle des esprits par le contrôle du passé. Ces commémorations ne tiennent pas compte, par ailleurs, de la chronologie, de la logique du temps.

7 Le lecteur, enfin, ne peut pas être surpris ou déstabilisé par les informations du quotidien puisqu'il a déjà une connaissance complète du contenu. La dernière décision prise par Tito est répétée. L'information est nulle, son contenu est connu et consensuel.

\section{L'évolution du culte}

8 Parmi la masse considérable d'informations disponibles, nous mettons l'accent sur quelques grandes évolutions de l'image de Tito le 25 mai. À partir de notre sélection, quatre grandes périodes peuvent être distinguées :

- de 1945 à 1955 : la fête du 25 mai célèbre le premier héros national yougoslave ;

- de 1956 à 1965 : le "jour de l'anniversaire de Tito" devient le "Jour de la Jeunesse" à sa demande, mais le gigantisme et la démesure de la célébration cachent mal un culte de la personnalité exacerbé ;

- de 1966 à 1974 : durant ces années de crises économique, sociale et nationale, le plan de propagande est modifié. La présence de Tito le 25 mai se fait plus discrète, les éloges moins appuyés ;

- de 1975 à 1980 : le stratégie de propagande, encore modifiée, met l'accent de nouveau sur le caractère hérö̈que du chef de l'État, et prépare les habitants de la Fédération socialiste yougoslave à l'après Tito.

\section{Le processus d'héroïsation du dirigeant yougoslave : 1945-1955}

La štafeta en l'honneur de Tito a été courue pour la première fois en 1945. Imaginée par un professeur et ses élèves, le départ de la course a été donné alors que le pays n'avait pas encore été totalement libéré de l'occupation allemande (avril 1945). Tito est né en 1892 et fêtait donc en 1945 son cinquante troisième anniversaire. La caractéristique essentielle du traitement journalistique de cet événement est de présenter Tito sous la forme d'un héros sans pareil. Des descriptions physiques et morales sont faites du maréchal Tito. Un ancien partisan, proche de Tito raconte à Politika sa première rencontre avec lui : «son visage m'est apparu attirant, ses traits étaient durs, preuves d'un caractère fort, et d'une grande énergie, (...) ses yeux étaient très clairs, son regard 
très doux et expressif, plein d'idéal et d'amour pour les hommes, sa voix chaude et sérieuse montre un souci constant de l'avenir des gens ; il a le front haut... » (25/05/45).

Cette description psychomorphologique met en avant le caractère très fort, très déterminé de Josip Broz Tito. La sollicitude que le dirigeant yougoslave exprime envers les gens est plusieurs fois soulignée dans la même phrase. Ce trait de caractère est encore évoqué par la suite: "Tito reçoit beaucoup de lettres, comme celle, par exemple, d'une petite fille de partisans, orpheline. Sa date de naissance a été fixée par les services sociaux au premier mai 1941. L'enfant n'ayant pas droit aux allocations familiales, Tito décide de lui donner dix mille dinars » (25/05/47).

11 La générosité et l'altruisme de Tito ont raison de la logique administrative (bien que le principe d'attribution de ces aides sociales ne soit pas remis en cause). Le journal Politika insiste, tout au long de ses articles, sur le sacrifice et l'abnégation de Josip Broz Tito pour les autres : «c'était le meilleur camarade au combat, il se souciait de tous les soldats au quotidien » $(25 / 05 / 47)$; « il y a avait un demi-agneau pour 40 personnes, Tito a partagé la nourriture et s'est servi le dernier » (26/05/52) (extraits de discussions entre anciens combattants).

12 Notons l'insistance sur le comportement exemplaire et courageux de Tito. Il réunit toutes les qualités humaines. Le chef de la Résistance est présenté comme "un héros", une personne qui se distingue par sa bravoure, ses mérites exceptionnels. "Tito est un héros populaire sans pareil » (25/05/45). Tito est un héros salvateur : « les ennemis ont mis à exécution leur plan prêt depuis longtemps ; (...) c'est à ce moment que Josip Broz Tito apparait. Son nom a traversé les peuples et les frontières » (25/05/46).

13 Au delà de l'homme parfait, irremplaçable, le maréchal Tito est une personne dont les capacités physiques sont décuplées par rapport aux autres; il est un surhomme, presqu'un héros herculéen, voire un demi-dieu : «idéologue du Parti, chef militaire, il est un organisateur et un leader des classes laborieuses dans la voie du socialisme. Il voit plus loin, il entend mieux que les autres » (25/05/52).

14 Le chef de la Résistance antinazie constitue une légende : sa vie fait l'objet d'un récit à caractère presque merveilleux, où les faits historiques se mêlent à l'imagination ou à la poésie. Progressivement, une légende s'inscrit à terme dans la mémoire collective et entre dans la tradition populaire. Tito est le premier héros national, c'est à dire qu'il est le premier héros commun à tous les habitants de la Fédération socialiste yougoslave, peuples culturellement très hétérogènes. Ces derniers ont désormais un mythe commun: leur dirigeant. L'histoire millénaire trouve son aboutissement avec l'action du maréchal yougoslave: "parler de Tito, c'est parler de l'étape la plus significative de l'Histoire de nos peuples » (extrait du discours de Bakarić, publié le 25/05/47).

Ses paroles sont découpées en prédications, en axiomes: «en novembre 1945, à Sarajevo, le camarade Tito avait dit: "je vous parle pour que vous sachiez la vérité, nous ne devons compter que sur nos forces" (...) ; Tito avait raison » (25/05/49 - extrait de l'article historique).

16 À l'omnipotence de Tito s'ajoute l'omniprésence. Nous en trouvons mention dans Politika: «on rencontre cette photo [de Tito] dans les maisons, les parcs, les bureaux, ses portraits sont admirés par des millions de personnes, Tito est un grand dirigeant, un chef militaire, un homme politique, (...), il est comme nous, sauf qu'il y a en lui tout ce qu'il y a de mieux (...)» $(25 / 05 / 53)$. 
17 Ces portraits ne sont pas sans rappeler les icônes. Un véritable lien d'amour unit Tito et les peuples de Yougoslavie : «dans les chants, (...) le peuple ne lui dit rien d'autre que son amour» $(25 / 05 / 45)$; «le nom de Tito est aimé par tous les Slaves » (25/05/46) (extraits de l'article historique).

\section{Un homme simple}

Outre un éloge appuyé de ses qualités humaines et morales, le milieu social et le mode de vie du maréchal yougoslave sont détaillés. Les origines sociales modestes de Josip Broz sont soulignées dans les articles historiques : «il courait pieds-nus à Kumrovec. (...) Tito n'a jamais baissé la tête » $(25 / 05 / 51)$; « Tito est né dans un des villages les plus pauvres de Croatie, il a grandi dans la pauvreté et a connu l'exploitation capitaliste. Le jeune Josip Broz est un ouvrier d'usine sans être un domestique » (25/05/52).

19 Les remarques faites sur les origines prolétariennes de Tito renforcent le caractère héroïque du personnage. Il n'est pas né héros, il l'est devenu. De nombreux détails sont donnés sur la vie de Tito, comme par exemple, dans un article consacré à une journée de travail du dirigeant yougoslave où ses moments de loisirs et de détente sont scrupuleusement décrits: "le rideau a tremblé et a découvert Tito. Il fait une promenade dans ses jardins à sept heures du matin. (...), il déjeune vers $12 \mathrm{~h} 30,(. .$.$) pour$ entretenir sa forme, Tito fait de la gymnastique et de la chasse, (...) le soir, Tito joue du piano ou fait une partie de billard ou d'échecs avec des amis » (25/05/52).

Des aspects de la vie privée et publique de Tito sont donc juxtaposés. Politika publie à la une, le 25 mai 1953, une photo représentant Tito durant ses loisirs. La légende est « le président Tito en promenade avec son fils ». L'image d'un homme resté simple, d'un père de famille attentif, est renvoyée au lecteur. La dichotomie homme modeste, "normal" et héros divin fonctionne selon la logique du balancier.

21 Le culte de la personnalité présente ici un dosage entre la déification et la simplicité de Tito. L'absence de délimitation entre la vie privée et publique est un phénomène fréquemment observé chez les personnalités publiques très médiatisées ${ }^{8}$.

\section{Un homme d'État exceptionnel}

22 La cérémonie de remise du relais au chef de l'État, place de la République à Belgrade, donne à ce dernier l'occasion d'aborder des points de politique générale, et, notamment, le développement économique. Il le fait sur un ton optimiste, encourageant, motivant pour la population: "c'est [la štafeta] la plus grande récompense que peut recevoir un homme. La štafeta est le symbole de la confiance, c'est un encouragement dans la poursuite de la construction du socialisme et du plan quinquennal » (26/05/48 - extrait du discours de Tito).

Tito est le moteur de la dynamique, de la motivation de la reconstruction. La réalisation du premier plan quinquennal doit être le seul but de tous les travailleurs yougoslaves. Il devait privilégier l'industrie lourde, selon une planification extrêmement volontariste. En mars 1946, une circulaire avait condamné toute entreprise de reconstruction qui n'était pas prévue par le plan. Ce plan quinquennal très ambitieux est réalisé (avec un peu de retard) grâce notamment aux millions d'heures de travail bénévoles. La politique d'information du quotidien joue un rôle essentiel de propagande, puisqu'au delà de la restitution de discours de Tito, Politika diffuse un message semblable sous 
d'autres formes. La réalisation des objectifs du premier plan quinquennal est encouragée et fonctionne en écho aux propos du dirigeant yougoslave :

de la part du président du gouvernement de la République Populaire de Macédoine, M. Koliševski, (...) nous mettons toutes nos forces dans le combat pour la réalisation et le dépassement du plan quinquennal; chaque homme de notre République populaire est touché par vos paroles et met tout en œuvre pour atteindre les objectifs... (25/05/47 - extrait d'un télégramme envoyé au Maréchal) ;

Avec la fin de la guerre, nous avons commencé la réalisation du plan quinquennal sous la direction de Tito (25/05/48 - extrait de l'article historique paru pour le cinquante-sixième anniversaire de Tito).

Les sujets polémiques (comme le conflit avec Staline) sont exclusivement traités par les journalistes de Politika. Le quotidien belgradois diffuse des messages de propagande concernant des points d'opinion pour faire pression sur les Yougoslaves. Tito, lui, ne s'exprime pas sur ces questions dans les colonnes du journal. Le traitement journalistique du règlement des litiges territoriaux entre la Yougoslavie et l'Italie à propos de l'Istrie ${ }^{9}$ et de Trieste est particulièrement significatif à ce sujet. Politika publie entre 1946 et 1954, des articles témoignant de l'attachement affectif des habitants de l'Istrie et de Trieste à la Yougoslavie par l'intermédiaire de télégrammes envoyés à Tito pour son anniversaire ou par des délégations venues spécialement le rencontrer ce jour là :

Cher Tito, de la part de l'Istrie slovène qui se bat pour son rattachement à la Yougoslavie, (...) chaque caillou, chaque arbre, chaque maison, chaque coin de l'Istrie s'expriment en deux mots : TITO et YOUGOSLAVIE. (...)

Vive notre guide le maréchal Tito, notre guide,

Vive l'Istrie slovène au sein de la République Fédérale de Yougoslavie,

Mort au fascisme,

Vive la liberté des peuples (25/05/46 - extrait du télégramme envoyé par "la jeunesse d'Istrie" au camarade Tito)

Le rattachement de l'Istrie et de Trieste à la Yougoslavie est présenté comme une aspiration du peuple lui-même; la poussée irrédentiste des néofascistes italiens est condamnée. Il s'agit, ici, d'une propagande suggestive, renforcée par la discrétion de Tito sur le sujet. Il est, par ailleurs, question plusieurs fois dans les journaux des 25 et 26 mai des nationalismes de façon implicite. Il est exposé de façon explicite que seul Tito défend le bon patriotisme :

LE $56^{\text {ème }}$ ANNIVERSAIRE DU MARÉCHAL TITO

LE CAMARADE TITO, INVENTEUR DU PATRIOTISME YOUGOSLAVE ${ }^{10}$

En s'inspirant de l'enseignement de Staline ${ }^{11}$ sur les questions nationales, Tito a fondé le patriotisme yougoslave et la fraternité et l'unité entre les peuples de la Yougoslavie populaire. (...) Le patriotisme veut que l'on respecte les autres peuples, il est étranger à tout chauvinisme, il est pour un profond Internationalisme. (25/05/48 - extrait de l'article historique.)

Le patriotisme de Josip Broz Tito a pour but de rallier la population à cette forme de nationalisme et de l'écarter des autres courants nationalistes plus radicaux et particularistes. Tito défend le nationalisme yougoslave contre celui d'une nationalité. 


\section{La fête nationale de la jeneusse célèbre Tito : 1956-1965}

\section{De l'anniversaire de Tito au Jour de la Jeunesse}

1956 est une année charnière dans la mesure où elle marque la fin de la célébration nationale et officielle de l'anniversaire de Tito et la première fête de la jeunesse (Dan Mladosti). Il est vrai que les participants à la course de relais étaient des jeunes gens ; plusieurs hommages leur ont été rendu pour leur action durant la Seconde Guerre mondiale, avant même 1956. Une certaine cohérence a donc été préservée.

Aucune explication officielle n'est fournie dans le numéro du 25 mai et la tribune ne précise pas l'appellation donnée à cette journée, bien qu'une allusion soit faite au vingtième congrès du PC Soviétique : «le développement économique et culturel a pris sous Staline des formes insupportables. Le vingtième congrès du Parti Communiste Soviétique a confirmé que le stalinisme ne pouvait survivre à Staline. (...) Les formes et les méthodes sont enterrées » (25/05/1956).

Ce changement est expliqué comme étant à l'initiative de Tito :

À la remise des cadeaux, Tito a dit : «chers camarades, merci pour cette dixième štafeta (...) Si ce jour est marqué comme étant celui de mon anniversaire, je pense qu'on devrait lui donner un autre nom : le jour de la jeunesse, le jour du sport, le jour de la nouvelle génération ».

La fin du discours de Tito a été saluée par des applaudissements au cri de «Tito, Tito ». (26/05/56)

"Chers camarades, merci à vous et à travers vous, les peuples de Yougoslavie, l'armée et les collectifs de citoyens. (...) Pour finir, je voudrais que le jour du 25 mai soit toujours le jour de la revue (smotra) de la jeunesse yougoslave et du sport, le jour de la revue du corps et de l'esprit.

VIVE NOTRE JEUNESSE et LES PEUPLES DE NOTRE PATRIE SOCIALISTE ! » (26/05/56)

30 Le journal Politika du 26 mai 1956 transcrit le discours de Tito au style direct : c'est le Maréchal de Yougoslavie qui justifie cette modification d'appellation en s'adressant directement à la population (et aux lecteurs de Politika).

Ce changement est peut-être lié au contexte politique de l'époque : 1956 est l'année où le culte de la personnalité de Staline est aboli en Union Soviétique, lors du vingtième congrès du PC Soviétique en février. Le vingtième congrès du PC Soviétique renvoie la responsabilité du conflit de 1948 à Staline, conflit qui avait eu pour conséquences l'exclusion de la Yougoslavie du Kominform en juin de cette même année et la rupture des relations diplomatiques entre les deux pays. Autrement dit, d'après le rapport de Khrouchtchev, Tito s'est opposé avec raison au despotisme de Staline. Par ailleurs, le culte de la personnalité introduit de son vivant par Joseph Staline est mis en cause : dans la partie neuf du rapport secret publié par le quotidien français Le Monde en juin 1956, les corrections des publications sur Staline par l'intéressé sont dénoncées, l'instauration de Prix Staline est critiqué : «les tsars eux mêmes n'ont jamais créé de prix portant leur nom ». A contrario, Politika souligne que le président Tito est "une légende car il s'est gardé d'en devenir une » (25/05/61). La déclaration de Tito a donc pour but de se démarquer du dictateur soviétique, qui après avoir été officiellement un modèle devient un repoussoir. La nature de cette fête a-telle changé pour autant? 
Politika s'applique à décrire de façon très détaillée le déroulement des spectacles du 25
mai. En ce qui concerne les célébrations elles-mêmes, le rassemblement final ne se fait
plus Place de la République mais au stade JNA, devant 50000 spectateurs en moyenne ;

Politika s'applique à décrire de façon très détaillée le déroulement des spectacles du 25
mai. En ce qui concerne les célébrations elles-mêmes, le rassemblement final ne se fait
plus Place de la République mais au stade JNA, devant 50000 spectateurs en moyenne ;

L'imputation des torts à Staline dans la rupture de 1948, la stigmatisation des extravagances du culte du "Petit Père des Peuples" influencent, semble-t-il, celui de Tito car c'est à partir de ce changement d'appellation que la fête du 25 mai devient grandiose, gigantesque et, paradoxalement, elle n'est pas sans rappeler la parade des sports soviétique. L'abolition du culte de la personnalité n'est donc qu'une déclaration d'intention. En effet, l'examen des faits contredit nettement la fin du culte de la personnalité en Yougoslavie.

Un exemple illustre parfaitement l'enchevêtrement de symboles liant le "Petit Père des Peuples" au "Vieux" : la formation de la première brigade prolétarienne yougoslave fut déclarée le 21 décembre 1941, jour de l'anniversaire de Staline. Cette date est devenue le jour de la fête de l'Armée Populaire de Yougoslavie après la guerre et ne fut modifiée ni par la rupture de 1948, ni par l'abolition du culte de Staline en février 1956.

Paradoxalement, le nom donné à cette fête nationale n'est pas fixé de façon définitive en 1956, malgré le vœu officiel de Tito. La comparaison des gros titres de la tribune montre l'inconstance de la désignation de cette journée ; en effet, ce n'est qu'à partir de 1965 -soit presque dix ans après le discours de Tito justifiant le changement- que la dénomination "Jour de la Jeunesse" est employée sans référence à Tito. La transition de l'un à l'autre se fait progressivement.

«L'anniversaire de Tito et le Jour de la Jeunesse » (25/05/60)

"À l'anniversaire de Tito et au Jour de la Jeunesse » (25/05/61)

«Fête du 70ème anniversaire de Tito et du Jour de la Jeunesse » (25/05/62)

« Jour de la Jeunesse et anniversaire du Président Tito » (25/05/63)

" Anniversaire de Tito et jour de la jeunesse » (25/05/64)

« 20 ans de fête du 25/05, la fête de la Jeunesse » (25/05/65)

La même date a été conservée pour la célébration de ces deux événements commémoratifs distincts. Alors que le 25 mai célèbre désormais officiellement la jeunesse, le quotidien belgradois continue de publier les télégrammes de félicitations que le président de la République reçoit pour son anniversaire et "la Charte de la Jeunesse et des peuples de Yougoslavie", c'est-à-dire le message annuel de congratulations destiné au maréchal Tito. Josip Broz Tito est pleinement associé à la fête de la Jeunesse et cela est perceptible jusque dans le choix des photographies illustrant les articles de journaux: il n'y a plus désormais de portraits figés pris en intérieur, sur un fond sombre. Les photos publiées à la une sont toujours prises en extérieur. Le président de la République ne pose plus, il est accompagné de jeunes gens. Par ailleurs, il abandonne son air grave pour une attitude plus détendue et un air plus gai, il est toujours souriant sur les photos. Un ton plus léger, plus joyeux est conféré à cette fête. Ces représentations très contrastées présentent par ailleurs certainement un intérêt nouveau des foules pour cet événement.

Des timbres-poste continuent, par ailleurs, à être émis pour les anniversaires de Josip Broz Tito sans référence au Jour de la Jeunesse (en 1962, 1967, 1972, 1977). Ce changement d'appellation ne marque donc pas, malgré les apparences, la fin de la célébration de l'anniversaire du président yougoslave.

\section{Une fête grandiose et ritualisée}

Balkanologie, Vol. III, nº 1 | 1999 
il n'y a désormais plus qu'un seul témoin de la štafeta. L'association de l'Armée traduitelle un poids croissant dans le régime? Ces spectacles présentent au public des démonstrations sportives et des exhibitions de danse par des enfants, des adolescents, des jeunes gens de toutes les nations et nationalités, des soldats et des jeunes travailleurs devant une foule nombreuse décrite comme enthousiaste, passionnée.

Le spectacle est soigneusement mis en scène et répété par les participants venus de tout le pays, souligne Politika. Le spectacle semble donner de la joie, du bonheur à ceux qui y assistent. Voici des extraits de reportage illustrant l'unanimité des spectateurs et des journalistes quant à la beauté du spectacle : «le spectacle dans le stade est une véritable symphonie de mouvements, de couleurs et de musique»(26/05/62) ; "l'enthousiasme a encore grandi quand les porteurs de la štafeta sont entrés sur le stade, avec les Vœux des Peuples et de la Jeunesse au président Tito pour son anniversaire » (25/05/64).

Les spectateurs attendent avec impatience Tito, dont l'entrée est annoncée par des fanfares. C'est donc un spectacle à la fois visuel et sonore, la musique ayant un côté exaltant et cohésif : «les fanfares constituent un signe que tout le monde connait : le camarade Tito arrive » (26/05/63 - extrait de la description du spectacle).

La musique des fanfares constitue ce que Tchakhotine nomme « un signal déclenchant du réflexe conditionné $»^{12}$. Les mécanismes du système nerveux sont déclenchés au moment voulu afin d'obtenir un comportement déterminé, qui s'apparente au dressage.

Le champ lexical de la religion est employé dans la description et l'analyse des célébrations du 25 mai. Les spectacles au stade de la JNA, ainsi que les rencontres à Beli Dvor ont un caractère protocolaire qui rappelle le "rituel". Les manifestations du 25 mai revêtent un caractère sacré, immuable et répétitif, comparable au rite (c'est-à-dire à un ensemble de gestes, prières, règles et cérémonies qui se pratiquent dans une Église particulière ou dans une communauté religieuse). Le déroulement de la journée est sans surprise et consensuel; il appelle un comportement conformiste comme dans la liturgie. On attend de l'individu au mieux du zèle, sinon de la passivité. Le 25 mai est une journée de culte rendu à Tito, dans le sens religieux du terme. C'est-à-dire l'hommage rendu à Dieu (adoration) ou à un Saint (vénération) par des gestes et des paroles exprimant une attitude intérieure.

\section{Un lien affectif intact unit les Yougoslaves à Tito...}

Politika souligne à maintes reprises le lien très fort unissant Tito aux Yougoslaves :

C'est un jour que nous aimons car nous aimons l'homme qui est lié à tout ce qui s'est passé et à tout ce qui va arriver (...) nous voulons te dire l'expression de notre amour (...) qui croît chaque jour (25/05/63) ;

Cher camarade Tito, nous t'apportons nos vœux chaleureux ; l'amour que chacun de nous te porte est l'expression de notre unité... (25/05/65) (extraits de la Charte des Peuples et de la Jeunesse de Yougoslavie).

L'expression de l'affection, voire de tendresse, envers le dirigeant yougoslave est sans équivoque, et rappelle la prière d'action de grâce, c'est-à-dire de remerciements. L'attachement à sa personne est également exprimé par les cadeaux qu'il reçoit. En 1962, Tito est élu citoyen d'honneur de toutes les mairies; à cette occasion, il reçoit des présents qui, d'après le journaliste, sont "le symbole de la valeur nationale" 
(tapisseries, documents datant de la guerre, qui sont un remède "contre l'oubli"). Voici d'autres exemples de cadeaux que Tito a reçu :

du matériel de chasse, des meubles de jardin en aluminium de Skopje, les Slovènes lui ont offert un tableau le représentant en ouvrier métallurgiste en 1911, la Maison de la culture de Belgrade la collection des cinq volumes des discours qu'il a prononcés... (25/05/61).

Les représentants du Monténégro lui ont offert des fleurs rouges,(...), les Croates une photo de Šišak, où était situé son atelier d'apprentissage ; les Macédoniens un plat du XVII ème siècle et une nappe brodée, (...), un album de photos sur la construction d'un hydrosystème, (...) les délégués de Bosnie-Herzégovine une cafetière et une lampe de chevet en mosaïque, l'Union des retraités, un album pour le quarantième anniversaire du journal Komunist. (25/05/65)

Ces cadeaux, signes d'affection exprimés par ses collaborateurs, par des représentants institutionnels, ainsi que par des délégations de citoyens, peuvent être regroupés en quatre catégories principales :

* des présents destinés à l'usage personnel (matériel de chasse, cafetière ou antiquités d'Art);

* des souvenirs de la jeunesse de Josip Broz (le tableau et la photo);

* des symboles de l'appartenance communiste du dirigeant yougoslave (les fleurs rouges et l'album);

* enfin, la représentation des succès réalisés sous le gouvernement de Tito.

Ces cadeaux ont été regroupés dans un musée inauguré le 25 mai 1962 à Belgrade ${ }^{13}$, près du Hajd Park. Politika consacre plusieurs pages à cet événement.

L'héroïsme de Tito n'est plus mis en avant. En effet, en 1962, le dirigeant yougoslave n'est pas élu Héros National, mais Citoyen d'honneur de toutes les communes de Yougoslavie. Il reçoit, à cette occasion, une charte - intégralement publiée par Politika lui exprimant toute la reconnaissance des peuples de la communauté des Slaves du sud pour son action politique exemplaire. En effet, Tito n'est plus un héros mais « Tito est (...) l'humble fils et le dirigeant politique le plus aimé de chacun de nos peuples » (Une du 25/05/62). Le personnage de Tito n'est plus empreint de merveilleux, mais au contraire de réalisme. C'est donc avec pragmatisme qu'il aborde les questions de développement. Ses capacités de réaction, d'innovation sont mises en relief.

\section{...malgré les difficultés économiques et sociales du pays}

Le dirigeant yougoslave a déjà discouru sur les questions économiques, mais toujours dans le sens des encouragements et de la motivation. Les discours de Tito concernent maintenant "les faiblesses de [notre] développement" :

Je sais que notre développement est jonché de difficultés; par exemple, les consommateurs souhaitent un prix raisonnable des produits. Un internédiaire intervient entre vous, les collectifs de travail et les consommateurs, et fait monter les prix ; les travailleurs ne profitent pas de la totalité des fruits de leur travail. Cela porte préjudice à la consommation et à la production... (25/05/61 - extrait du discours de Tito aux représentants des collectifs de travail à la réception de Beli Dvor).

Il faut d'abord nous soucier des travailleurs qui sont le moins payés. Nous devons diminuer les différences de salaire entre les travailleurs. (...) Nous devons reporter la construction des immeubles qui ne nous sont pas utiles immédiatement. (...) Je suis sûr que les travailleurs comprennent nos difficultés, il n'est pas difficile de les convaincre. (...) Nous ne devons construire que des usines rentables et améliorer 
celles qui existent déjà. (25/05/62 - extrait d'un discours de Tito lors d'une rencontre avec les responsables des collectifs de travail).

Dix ans après la loi sur l'autogestion, le dirigeant yougoslave évoque, pour la première fois, les difficultés traversées par le pays. Le maréchal yougoslave se présente comme le seul détenteur des réponses aux difficultés traversées par le pays. Tito est un guide intellectuel et moral. C'est un des ressorts essentiels, nous semble-t-il, du culte de la personnalité du "Vieux". Le culte de Tito a évolué, il est plus réaliste, moins maladroit.

\section{Un exemple de modification de la stratégie de propagande : une présence plus discrète dans un contexte d'agitation sociale et nationale (1966 -1974)}

\section{Une modification de la stratégie de propagande}

49 À partir de 1966, et contrairement aux années précédentes, des articles concernant d'autres sujets que ceux habituellement traités sont publiés à la une des 25 et 26 mai. Il ressort d'une rapide analyse quantitative que $70 \%$ des unes sont consacrées exclusivement à Tito ou au Jour de la Jeunesse dans les journaux du 25 mai de 1945 à 1980. Cependant, de 1966 à 1974, soit 9 années consécutives, les unes ne sont pas entièrement consacrées à Tito ou au Jour de la Jeunesse, avec un minimum de $18 \%$ en 1971, alors que le président de la République fête ses 80 ans.

Ce sont essentiellement les relations internationales, (guerre du Viêtnam, la rencontre soviéto-américaine Brejnev-Nixon) et la politique extérieure de la Yougoslavie (relations avec l'Égypte) qui occupent le reste de la surface des unes de 1966 à 1974. De surcroît, la photo du Maréchal de Yougoslavie n'est pas en couverture des journaux du 25 mai des années 1969, 1971, 1973.

51 La diminution de la part des articles consacrée à Tito et au Jour de la Jeunesse correspond-elle à un changement de plan de campagne (stratégie) ou à une inflexion de la propagande titiste? Nous n'avons que peu d'éléments de réponse ; cependant, $70 \%$ du total des émissions philatéliques à l'effigie du maréchal ont lieu au cours de cette même période. Toutefois, la hiérarchie de l'information est nettement modifiée, c'est-àdire que la politique d'information du journal octroie une part plus importante à la politique internationale et "délaisse" le "25 mai". L'internationalisation de l'information et le succès du mouvement des non-alignés sont également des hypothèses pouvant expliquer le phénomène. À partir de 1968 et ce jusqu'en 1970, l'expression "Jour de la Jeunesse" est exclusivement employée, c'est-à-dire pendant les périodes de reconnaissance d'un semi-échec. En 1971, il est uniquement question de l'“anniversaire du président Tito".

\section{Un aveu de semi-échec}

La Yougoslavie traverse, à partir du milieu des années 1960, une crise constitutionnelle, économique et sociale, qui se double en 1966 d'une crise politico-judiciaire avec la destitution du chef serbe de la police Aleksandar Ranković. En 1965, en effet, la Constitution yougoslave a été modifiée : les fonds sociaux d'investissement pour les régions les moins développées sont transférés aux banques qui doivent les gérer selon des critères économiques. La planification n'a donc plus qu'un rôle indicatif. Les prix 
intérieurs sont ajustés sur les prix mondiaux, ce qui entraîne une envolée de l'inflation ( $30 \%$ en 1965 et $14 \%$ sur la fin de la décennie, contre $7 \%$ pour la période 1960-1964) et la dépréciation de la monnaie. Le chômage augmente et la dette gonfle artificiellement. Les nationalistes s'agitent, le mécontentement des ouvriers et des étudiants monte. Les grèves sont niées ou condamnées, bien que de nombreux mouvements d'“arrêts de travail", selon la formule officielle, aient été comptabilisés ${ }^{14}$.

La principale revendication portait sur les salaires. Ce sont les Républiques les plus développées qui ont connu le plus de conflits de travail. Ces grèves n'ont pas débouché sur l'explosion, car elles ont été localement très efficaces, puisque les ouvriers avaient pour eux d'être les maîtres de l'usine, dans le Droit et dans l'idéologie dominante, c'est le principe de l'Autogestion. ${ }^{15}$

Parallèlement, un mouvement étudiant, né en juin 1968, dénonçait outre l'intervention de l'armée américaine au Viêtnam, "la désintégration de la propriété sociale, le chômage, les inégalités sociales, la commercialisation de la culture ${ }^{16}$, l'extension de la propriété privée tolérée par la réforme constitutionnelle de 1965. Plusieurs universités furent occupées. C'est dans ce contexte que, pour la première fois, Tito fait un constat de semi-échec:

Nous nous trouvons encore à une période agitée de notre développement socialiste. (...) Nous avons travaillé à la reconstruction, mais parfois trop lentement. (...) Parfois, nous n'avons pas fait ce qu'il fallait (25/05/66).

Nous avons déjà répété que la jeunesse est notre plus grande richesse. Je dois cependant dire que nous n'avons pas réalisé toutes nos promesses. (...) Il faut continuer à intégrer notre jeunesse dans nos usines, écoles et universités (26/05/68).

Il s'agit d'un aveu des fautes commises, aveu atténué par l'adverbe "parfois" et par l'utilisation systématique de la première personne du pluriel.

Les articles historiques reprennent également l'idée de ces échecs : «reconnaissance des faiblesses du système scolaire » $(25 / 05 / 66)$; « le monde dans lequel nous vivons est loin d'être bon, il y a de quoi faire » $(25 / 05 / 67)$; « nous n'avons pas réalisé toutes nos promesses, nous devons aller plus vite » $(26 / 05 / 68)$.

D'après Politika, la responsabilité de chaque citoyen est engagée. C'est une preuve du soutien inconditionnel accordé au dirigeant yougoslave. C'est un tournant dans le culte de la personnalité de Tito, car les champs sémantiques relatifs à l'héroïsme ont disparu. Les qualités d'“homme politique d'expérience" et de conviction suppléent celles du "héros sur-humain" empreint de merveilleux. Le rapport social entre les Yougoslaves et Tito est, d'après l'auteur de l'article, modifié: «nous, les contemporains du camarade Tito, nous sentons comme ses collaborateurs » (25/05/67).

57 Les Yougoslaves sont associés au pouvoir, car un rapport de proximité est instauré. C'est un contre-pied à la stratégie de culte précédente puisque la nature des rapports entre les Yougoslaves et leur président de la République est évoquée autrement que par des relations "transcendantes". Tito se veut donc à l'écoute de la jeune génération :

"Je voudrais leur (aux jeunes) dire que je vais faire, comme je l'ai fait dans le passé, tout ce que je peux dans la limite de mes forces, pour le bien de notre Union". Le discours de Tito a été entrecoupé d'applaudissements (25/05/66 - élocution du chef de l'État yougoslave et commentaire de Politika).

Une jeune fille dit que l'on n'écoute pas assez ce que les jeunes proposent. Tito s'adressant à elle : «il faut parler ouvertement, ne vous gênez pas, la critique est une chose, la censure en est une autre » (25/05/66). 
Politika insiste sur le fait que Tito est soucieux des préoccupations quotidiennes de ses concitoyens :

Tito a demandé des nouvelles du tremblement de terre de Banja Luka. (Une femme l'informe.) Ça va de mieux en mieux (...) ; tout le monde donne le maximum de soi.

- Il faudra encore du temps, du travail et de la patience mais on y arrivera, a répondu Tito.

- Y a-t-il de la neige au Monténégro ? demande Tito.

- Comme d'habitude, lui répond-on.

(...) Les Slovènes l'interrogent sur le sport : quelle équipe soutenez-vous?

- J'ai d'abord soutenu Partizan, mais maintenant aucune, car on surveillait l'équipe que j'applaudissais. Je soutiens les équipes yougoslaves dans les rencontres internationales (25/05/70).

Le Maréchal de Yougoslavie réaffirme son investissement personnel pour le bien-être de ses compatriotes : « chaque rencontre avec les citoyens m'apporte un nouvel élan ; je dois donner encore plus de moi-même » (25/05/69).

\section{Les attentes des Yougoslaves réalisées}

Les réformes de 1965 ont entraîné des difficultés économiques accrues et des tensions entre nationalités. Aucun nom n'est cité, aucun point concret n'est développé par le journal Politika. Sans polémiquer, Tito intervient en personne, prononce un discours de régulation, d'arbitrage, au sens propre des deux expressions, c'est-à-dire de corrections de fonctionnement, de règlements et d'apaisement des litiges. Ses messages se veulent également rassurants pour la population: les difficultés sont passagères, les succès durables, certaines revendications satisfaites.

Notre Fédération Socialiste est composée de nations et nationalités, (...) elles ont vécu séparées, dans des conditions différentes mais difficiles. Notre combat a rendu possible leur union dans un même pays. Chaque nation et nationalité y a toutes les libertés et la possibilité de s'autogérer. Vous voyez que nous faisons des amendements constitutionnels pour que chaque république ait encore plus de droits et de devoirs; ces droits et devoirs incombaient jusqu'à présent à la fédération (26/05/71 - extrait du discours de Tito publié en première page $d u$ journal, dont la une est «n'oubliez pas de défendre et de renforcer l'unité de nos peuples »).

61 Tito réaffirme sa position sur des questions d'opinion, c'est-à-dire qu'il tente d'exercer une influence, notamment sur des questions d'actualité :

«(...) Il y a des gens qui pensent que les rapports entre les nationalités sont historiquement déterminés. (...) La société socialiste n'annule pas les nationalités, leur origine et leurs réalisations dans le passé. Mais dans le socialisme, les désaccords nationaux doivent disparaître. Je vous remercie d'être venus, vous êtes ici chez vous. " Les jeunes ont salué le discours de Tito par des applaudissements sincères. (26/05/71)

62 La crainte d'une alliance entre mouvement ouvrier et étudiant poussa Tito à donner satisfaction à certaines revendications de la "nouvelle gauche" (regroupée autour de la revue Praxis) : la propriété privée fut limitée. En 1972, quelques mois après la fronde nationaliste, Tito reçoit le second Ordre du Héros National. Cette décoration marque les prémices d'un nouveau tournant dans la stratégie du culte du dirigeant yougoslave ; la crise, souvent qualifiée de "printemps croate", avait des causes multiples, d'après J. Krulic :

- une racine économique puisque les partisans d'un système bancaire décentralisé et les 
défenseurs d'une redistribution des ressources par les banques belgradoises s'opposaient sur la question de la solidarité économique entre les républiques de la Fédération yougoslave ;

- une cause linguistique puisque le débat, longtemps étouffé, sur les différences entre la langue parlée par les Croates et celle parlée par les Serbes était de nouveau posé ;

- une "cause identitaire", puisque certains nationalistes rejetaient toute responsabilité du peuple croate dans les événements de $1941^{17}$.

\section{Le retour à l'héroïsation, la préparation de la succession : 1975-1980}

63 À partir de 1975, le Jour de la jeunesse revient au sommet de la hiérarchie de l'information; $100 \%$ de la surface de la Une est consacré au Jour de la Jeunesse. Politika met en place une tactique de propagande pour soutenir le projet de Tito qui prévoit son élection à la présidence à vie. Une nouvelle constitution, qui tente de renationaliser le système bancaire, est promulguée en février 1974. L'autonomie des banques est réduite ; cette réforme est donc, en partie du moins, en opposition avec les réformes de 1965. L'autogestion est généralisée, mais l'autonomie des acteurs sociaux des entreprises est réduite. La nouvelle constitution accroit les prérogatives des républiques au dépens du centre fédéral, notamment en matière économique.

\section{Reprise de l'image du héros}

L'image du héros est employée avec beaucoup d'insistance :

C'est une légende vivante. Tito est un grand héros (26/05/76 - propos de jeunes gens recueillis par un journaliste de Politika quelques heures avant le spectacle au stade de la JNA) ;

Tito, triple héros - (...) Tito est l'homme de la Révolution, il est héroïque, mais son héroïsme n'est pas lié à des situations exceptionnelles, c'est un mode de vie... (25/05/77 - article publié lors de la remise à Tito de l'Ordre du Héros National pour la troisième fois).

Un concours de poésie intitulé "la belle lettre T" est organisé, en 1977, par Politika pour les enfants et adolescents jusqu'en classe de troisième sur le thème "Tito". La rédaction précise que les plus beaux poèmes publiés ont été choisis parmi les 1000 reçus. Le président Tito incarne de nouveau un personnage légendaire puisqu'il fait l'objet de poésie. L'un des poèmes publiés, «Merci, Tito ", rappelle les prières d'action de grâce. Si Tito est un héros, la jeunesse, à qui s'adresse officiellement la fête du 25 mai, n'en est pas moins héroïque : «la jeunesse est un lien avec notre passé héroïque » (extrait du discours de Tito - 25/05/75).

Au delà de l'image du héros, la figure de Tito est également associée à celle d'un sage ; Tito fait toujours preuve de sûreté dans ses jugements et sa conduite. La logique du balancier entre l'homme commun et le héros est reprise. "Tito et nous, on se comprend, (...) c'est un ami » $(25 / 05 / 76)$; « Tito est génial car il sait que l'attachement à un bloc augmente le danger de guerre » (25/05/77).

67 L'accent est mis sur l'élégance de Tito en 1979 : « doyen de la scène politique, [il parle] au dessus du niveau standard de la diplomatie, c'est le style Tito » (25/05/79) ; « Tito est le dirigeant le plus distingué du monde d'aujourd'hui » (25/05/79). 
Tito se distingue par son comportement élégant, par son bon goût. Le Président de la République est un personnage charismatique, qui possède l'art du discours. Il est présenté comme un personnage historique :

«Un rôle historique » (sous titre de l'article historique - 25/05/77);

Tito a reçu en cadeau les quatre premiers volumes d'une oeuvre qui en comptera 50 ; les quatre premiers traitent de la vie de Tito de 1926 à 1939 (25/05/79 description et commentaires de la remise de l'Ordre du Héros National à Tito) ;

«Une responsabilité exceptionnelle devant l'Histoire » (titre de la une - 25/05/78).

Les paroles du dirigeant yougoslave sont découpées en sentences :

Tito a dit en 1946 : "il faut travailler plus..." (25/05/77);

Sur l'Hitlérisme, extraits des discours de Tito : "Ce n'est pas un bon voisin, il est un ennemi de la liberté et de l'indépendance des peuples de Yougoslavie", discours (...) écrit en 1938 à Paris (25/05/77)

Tito a dit en 1946 : "Nous ne nous battons pas seulement pour nous mais aussi pour les générations futures" (25/05/78).

Un héros est par définition un bienfaiteur, c'est-à-dire une personne, qui, par ses actes de bonté, rend les gens heureux. C'est ainsi que de nombreuses inaugurations ont lieu le 25 mai comme celle de 80 logements sociaux en Macédoine en 1975 :

Le Jour de la Jeunesse et l'anniversaire du président Tito ont été fêtés dans tout le pays. De nombreux sites ont été inaugurés comme des routes, des écoles, des dispensaires. (...) Ce sont les victoires des travailleurs (26/05/75 - extrait du chapeau de la Une, impression en caractères gras);

Première pierre posée de la maison de la culture dans la ville natale de Tito "L'armée est en constante modernisation" a lancé le général Jovanović, (...) lors d'un meeting militaire (25/05/76);

«Ouverture d'une crèche pour 100 enfants le 23 mai; mise en fonctionnement le premier juillet » (25/05/76).

71 Alors que les infrastructures ne sont pas encore achevées ou mises en service, leur inauguration est anticipée afin de la faire correspondre à la célébration du 25 mai. Le nom de Tito est donc toujours connoté positivement, et associé à une amélioration, un progrès, une avancée sociale.

Josip Broz Tito est un homme compétent, réputé pour ses connaissances et son expérience, une personne dont les avis sont écoutés, et qui tend à réaliser un modèle idéal de vie :

Le courage de ton œuvre révolutionnaire (...) est pour nous le message d'une vie (25/05/75 - extrait de la lettre qui va être transmise à Tito avec l'Estafette) ;

Notre jeunesse a toujours eu un guide qui lui a montré comment défendre et aimer la paix, la liberté et l'indépendance (25/05/76).

L'idée du guide est reprise à nouveau. Ces extraits de Politika sont en opposition avec ceux de la période précédente qui concernent les rapports entretenus entre les peuples yougoslaves et Tito. Les habitants de la Fédération yougoslave ne sont plus traités en collaborateurs mais en exécutants.

\section{La question de la succession}

En 1975 (Tito a 83 ans), la question de la succession au pouvoir est posée par Tito luimême :

Qu'est ce qu'il y aura après Tito ? Cette question est sans fondement. Tito n'a pas

fait tout cela tout seul ; nous l'avons fait tous ensemble. (...) Le mérite en revient à 
tous. (...) Je suis Président de la République et président de la Ligue des communistes de Yougoslavie, mais qu'aurais-je pu faire sans les gens qui m'entourent? (25/05/75).

\section{à partir de 1976, le maréchal yougoslave est toujours représenté seul ou entouré de ses} collaborateurs, au milieu d'une assemblée officielle. Plus aucune photographie ne montre le président Tito en compagnie de jeunes gens, exception faite d'une photo publiée en 1976 représentant Tito en compagnie d'une petite fille. Après avoir associé l'image du maréchal Tito au "peuple" yougoslave durant la période 1965-1974, ce sont à présent les dirigeants yougoslaves qui posent auprès du président vieillissant. On peut déjà entrevoir là le passage d'une autocratie (un système politique dominé par un seul homme) à ce qui va devenir une polyarchie (un système politique caractérisé par la pluralité des centres de décisions).

77 Tito décède le 4 mai 1980, soit trois semaines avant son quatre-vingt-septième anniversaire. La nouvelle direction du pays, fidèle au précepte "après Tito, Tito" poursuit les célébrations du 25 mai. À cette occasion, de nombreuses inaugurations sont fêtées (un pont sur le Danube, une nouvelle hydrocentrale baptisée "Tito"), de l'argent est versé sous formes de dons pour la bourse Tito. L'image du héros, du guide, est gardée. Tito est "un titan", une personne d'une puissance extraordinaire. Il "est entré dans les fables populaires", son œuvre est "éternelle", des poèmes lui sont dédiés : "il ne te faut pas une légende, un amour véritable t'est suffisant". La "grande confiance" de Tito dans les jeunes est répétée. L'orientation du culte de Tito dans les dernières années de sa vie (personnage héroïque, légendaire...) est confirmée par les articles parus pour son premier anniversaire post-mortem.

L'image de Tito n'est pas monolithique. Voici quelques uns des attributs qui lui sont conférés : généreux, philanthrope, personnage légendaire, divin, issu d'un milieu social modeste, féru de travail, bon père de famille, humble, motivant, idéaliste, indifférent à la polémique, patriote, compétent, audacieux, réaliste, collaborateur du peuple, politicien avisé, internationaliste, guide, sage, personnage charismatique... Le culte de la personnalité évolue, s'adapte à l'actualité et à l'opinion publique. La stratégie de communication est modifiée, ajustée en fonction de la conjoncture. Par ailleurs, l'élection de Tito à la présidence à vie montre que le culte exacerbé de la personnalité $\mathrm{du}$ dirigeant yougoslave influence la vie politique du pays. Le culte de Tito et les orientations politiques sont donc en interaction. Le culte de la personnalité est donc bien "un outil du titisme" pour reprendre l'expression de J. Krulic ; c'est un instrument qui a pour but l'adhésion physique (participation aux manifestations du 25 mai) et idéologique (le titisme en tant que système et doctrine) des Yougoslaves. La sacralisation du pouvoir est un détournement de la fonction de serviteur des citoyens. Pliant ces derniers à une révérence religieuse, elle inspire à la fois crainte et respect. L'autorité hiératique représente un danger, car elle tend à transformer les compétences en pouvoir, elle donne à celui qui en use une supériorité "naturelle", 
presque transcendante. C. Rivière ${ }^{18}$ exprime avec justesse l'idée du culte : «le chef a un corps sain et puissant, il présente des signes d'élection, il a surmonté des épreuves initiatiques, il se manifeste comme le garant de l'ordre, de la paix, de la sécurité, mais il sait châtier de manière exemplaire les transgresseurs de normes ». Le pouvoir sacralisé de Tito permet tour à tour d'occulter les échecs économiques, de justifier un traitement national inégal, de légitimer le monopole du Parti communiste et la limitation des libertés individuelles. Ces différents éléments sont autant d'explication de l'escalade tragique qui a mené les peuples de Yougoslavie dans la guerre civile.

Incontestablement, au vu de la guerre civile qui a déchiré la Fédération yougoslave une décennie après la mort de Tito, les efforts d'éducation titiste se soldent par un échec tragique. S'interroger sur les responsabilités du conflit en ex-Yougoslavie pose donc la question d'une responsabilité de son prestigieux dirigeant. En fait, Tito n'a pas, de son vivant, instauré un réel équilibre interne. À sa mort, l'immobilisme et les luttes d'influence ont eu raison de la Yougoslavie, fragile édifice régulièrement secoué par les appétits des nationalistes de tous bords.

La Yougoslavie de Tito n'est certes pas l'Union Soviétique de Staline ou la Roumanie de N. Ceausescu, mais l'élargissement des libertés fondamentales prônée par Milovan Djilas et d'autres aurait été, selon nous, un des moyens d'éviter l'attisement des haines et le déchirement.

\section{NOTES}

1. Krulic (Joseph), Histoire de la Yougoslavie de 1945 à nos jours, Paris : Complexes, 1993, p. 161.

2. Un doute subsiste cependant concernant la date exacte de l'anniversaire du chef d'État yougoslave: J. Krulic avance la date du 7 mai 1892 et constate qu'«il s'est attribué ou laissé attribué deux dates de naissance ». Comment expliquer ce changement? Le 25 mai 1944, plusieurs unités d'élite nazies sont parachutées sur Drvar en Bosnie-Herzégovine, où Tito avait installé son quartier général. Dans La Yougoslavie de Tito de 1937 à 1956, Thomas Schreiber note qu'«Hitler espérait ainsi que la neutralisation de Tito lui permettrait enfin de décapiter le mouvement de résistance. Mais après des combats acharnés, les troupes aéroportées allemandes furent presque complètement anéanties ». Il est donc possible que Tito ait choisi de changer consciemment de date de naissance afin de rappeler à chacun de ses anniversaires son rôle durant la Seconde Guerre mondiale.

3. Domenach (Jean-Marie), La Propagande Politique, Paris : PUF, 1979.

4. Lois dégagées par ibid.

5. La Bibliothèque de documentation internationale contemporaine (BDIC) à Nanterre possède tous les numéros de Politika depuis 1945.

6. Agnès (Y.), Lire le Journal, Saint-Julien-du-Sault : F.P. Lobies, 1979.

7. Ellul (J.), Propagandes, Paris : Economica, 1991.

8. Morin (Edgar), Les Stars, Paris : Seuil, 1984.

9. L'Istrie est une presqu'île de l'Adriatique; ancienne possession autrichienne, ce territoire devient la propriété des Italiens en 1920, de même que Trieste en 1919, d'où des tensions très fortes entre l'Italie et la Yougoslavie. En 1947, l'Istrie est un territoire libre, qui a pour capitale 
Trieste. Ce statut, fixé par le traité de Paris, est provisoire car les deux entités sont rattachées à l'Italie en 1954.

10. L'article est signé Vladimir Dedijer, rédacteur en chef du quotidien du parti Borba et proche collaborateur de Tito

11. Ne soyons pas surpris de cette référence à Staline à quelques semaines de l'exclusion de la Yougoslavie du Kominform (juin 1948) : "les communistes yougoslaves devaient prouver leur caractère authentiquement communiste (...), le culte de Staline est maintenu jusqu'au milieu de l'année 1949. Le cinquième congrès du PC yougoslave en juillet 1948 verra le spectacle surréaliste de Tito applaudi aux cris de "Tito, Staline" ( Krulic (Joseph), op.cit., p. 94).

12. Tchakhotine (S.), Le Viol des Foules par la Propagande Politique, Paris : Gallimard (Collection Tel), 1952.

13. Le musée du 25 mai était fermé en juillet 1998 et occupé par l'Armée, sans doute en raison des événements au Kosovo.

14. Samary (Catherine), Le Marché contre l'autogestion. L'expérience yougoslave, Montreuil / Paris : La Brèche / Publisud, 1988.

15. Ibid., p. 216.

16. Ibid., p. 217.

17. Krulic (Joseph), op.cit.

18. Rivière (Claude), Piette (A.), éds., Nouvelles Idoles, Nouveaux Mythes. Dérives de la Sacralité, Paris : L'Harmattan, 1990.

\section{RÉSUMÉS}

L'auteur analyse la fête du 25 mai telle qu'elle est présentée par le quotidien belgradois Politika. D'abord "anniversaire de Tito" puis "jour de la jeunesse", cette journée était l'occasion de nombreuses manifestations culturelles et sportives et la transcription qui en est faite par le quotidien sert à rendre compte de l'évolution de l'image de Tito. 